\title{
QUALIDADE DE FRUTOS DE PITANGUEIRA (EUGENIA UNIFLORA L.) DURANTE A MATURAÇÃO
}

\section{QUALITY ATTRIBUTES OF PITANGA FRUITS (EUGENIA UNIFLORA L.) DURING MATURATION}

\author{
Renato Lima Dantas ${ }^{\text {I* }}$; Eduardo Magno do Nascimento Bezerral"; Lindemberg Timóteo dos Santos II; \\ Pedro de Oliveira Alves ${ }^{\prime \prime}$; Lindojonio Pereira de Limall, Josiane Silva de OliveirallI
}

\begin{abstract}
Resumo. A pitangueira (Eugenia uniflora L.) pertence à família Myrtaceae, estando amplamente distribuída em países da América do Sul, principalmente no Brasil, seu centro de origem. Contudo, ainda são poucas as informações acerca dos aspectos da qualidade dos frutos apesar do seu elevado potencial. Na maturação, o fruto passa por mudanças acentuadas na coloração, resultado do acúmulo de pigmentos, como também mudanças físico-químicas que lhe conferem sabor e aroma atrativos para o consumo. Assim, este trabalho tem como objetivo avaliar as mudanças na qualidade de frutos de pitangueira durante a maturação. Frutos de pitangueira foram colhidos apresentando coloração da casca variando de verde amarelada até completamente vermelha. A colheita foi realizada na zona rural, Sítio Paripe, Conde-PB. Os frutos foram encaminhados para o Laboratório Multidisciplinar XI das Instituições Nova Esperança, João Pessoa-PB, sendo realizada a seleção e, com base na coloração da casca, feita a separação em três estádios de maturação. Para posterior análises físico-químicas de sólidos solúveis, $\mathrm{pH}$, acidez titulável e análise de coloração utilizando o aplicativo Colorimeter. Foi observado que a acidez dos frutos da pitangueira apresentou uma redução nos valores médios do estádio Amarelo Dourado para o Completamente Vermelho, de 3,1 para 2,3 g $100 \mathrm{~g}^{-1}$. Para o pH, foi observado que os frutos mais maduros tinham um pH mais elevado. Os sólidos solúveis (SS) apresentaram uma diferença significativa entre os estádios de maturação, observando-se um aumento de $\cong 30 \%$. Os parâmetros da coloração da casca pelo sistema CIELab avaliados pelo aplicativo mostraram que os frutos têm mudanças acentuadas ao longo de sua maturação. Desse modo, frutos de pitangueira vermelhos apresentam valores elevados para a acidez titulável e sólidos solúveis no estádio completamente vermelho. O mesmo ocorreu para o conteúdo de antocianinas e flavonoides amarelos.
\end{abstract}

PALAVRAS-CHAVE: Frutas Nativas. Gênero Eugenia. Índices de Qualidade. Antocianinas.

Abstract. Pitanga (Eugenia uniflora L.) belongs to the Myrtaceae family, being widely distributed in South American countries, mainly in Brazil, its center of origin. However, there is still little information about the quality aspects of its fruits, despite the high potential they have. During maturation, the fruit undergoes marked changes in color, because of the accumulation of pigments, as well as physicochemical changes that give to itself an attractive flavor and aroma for the consumption. Thus, this work aims to evaluate the changes in the quality of pitanga fruits during maturation. Fruits were harvested with peel color varying from yellowish green to completely red. The harvest was carried out in the rural area, Sítio Paripe, Conde-PB. The fruits were sent to the Multidisciplinary Laboratory XI of the Nova Esperança Institutions, João Pessoa-PB, where the selection was carried out and, based on the color of the peel, three stages of maturation were separated. For further physical-chemical analysis of soluble solids, pH, titratable acidity, and color analysis using the Colorimeter application. It was observed that the acidity of the pitanga fruits showed a reduction in the

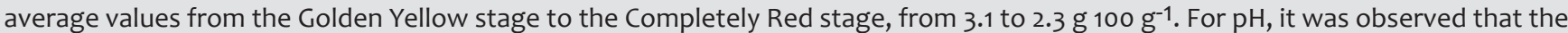
more mature fruits had a higher value. Soluble solids (SS) showed a significant difference between the maturation stages, where an increase of $\cong 30 \%$ was observed. The parameters of peel color by the CIELab system evaluated by the application showed that the fruits have marked changes throughout their maturation. Thus, red pitanga fruits show high values for titratable acidity and soluble solids in the completely red stage. The same occurred for the content of anthocyanins and yellow flavonoids.

KEYWORDS: Native Fruits. Eugenia Genus. Quality Indices. Anthocyanins.

IAgrônomo, Doutor em Agronomia (Fitotecnia/Fisiologia Pós-colheita). Docente Faculdade de Enfermagem Nova Esperança - FACENE. CEP: 58067-698. João Pessoa, Paraíba, Brasil. *Autor correspondente: renato_dantas@hotmail.com.br ORCID ID: 0000-0002-5464-9476.

"Graduandos em Agronomia, Faculdade de Enfermagem Nova Esperança. CEP: 58067-698, João Pessoa, Paraíba, Brasil. ORCID ID: 0000-0001-8164-9685; 0000-0002-0150-7140; 0000-0002-6882-7317; 0000-0003-2971-210.

III Química Industrial, Mestre em Engenharia de Processos. Docente Faculdade de Enfermagem Nova Esperança - FACENE. CEP: 58067-698, João Pessoa, Paraíba, Brasil. ORCID ID: 0000-0003-3640-0324 


\section{INTRODUÇÃO}

Frutas tropicais são mundialmente conhecidas e, tradicionalmente, apreciadas em função de características próprias de sabor e aparência. Contudo, é notória a importância que o consumo de frutas e hortaliças tem na promoção de saúde e qualidade de vida, especialmente em tempos de pandemia, em que a manutenção da imunidade do organismo é fundamental na mitigação dos riscos de doenças. As denominadas superfrutas são fontes, além dos nutrientes básicos para o funcionamento do corpo, de compostos responsáveis por diversas funções benéficas para o bom estado do organismo. ${ }^{1}$

A maioria das frutas nativas brasileiras é utilizada na produção de sucos, geleias e compotas de frutas, o que acontece para a pitanga também. Contudo, são fontes ricas de compostos bioativos de alta atividade antioxidante, com propriedades benéficas para a saúde e o consumo dessas frutas tem potencial para prevenir doenças crônicas não transmissíveis, como câncer, diabetes mellitus, dislipidemias, doenças cardiovasculares e doenças respiratórias crônicas. 2,3,4

A pitangueira (Eugenia uniflora L.), membro da família Myrtaceae, é nativa do Brasil e amplamente distribuída em países da América do Sul, como Argentina, Paraguai e Uruguai. ${ }^{5} \mathrm{O}$ fruto é uma baga globosa que, ao iniciar o processo de maturação, dependendo do genótipo, o epicarpo passa do verde para o amarelo, alaranjado, vermelho, vermelho-escuro, podendo apresentar o negro, resultado do acúmulo de pigmentos durante a maturação, como também acentuadas mudanças físico-químicas que conferem ao fruto sabor e aroma atrativos para o consumo. ${ }^{6}$ São reportados três tipos diferentes de pitanga conforme a cor do epicarpo do fruto: a pitanga-laranja, pitangavermelha e a pitanga-roxa. ${ }^{7}$

Devido à alta perecibilidade, os frutos da pitangueira são raramente encontrados no mercado na forma fresca, sendo o processamento a tecnologia mais empregada para seu aproveitamento. Geralmente, a alta perecibilidade se deve ao seu alto metabolismo durante o amadurecimento. ${ }^{8}$ Assim, o manejo inadequado nos períodos de colheita e pós-colheita acelera os processos de maturação e senescência. Conforme Vizzotto et al. ${ }^{9}$, o estádio de maturação em que o fruto é colhido determina a qualidade final para o consumidor. Os frutos colhidos verdes são inaceitáveis comercialmente, apresentam altas taxas de perda de água e são muito suscetíveis a distúrbios fisiológicos. Por outro lado, os frutos colhidos muito maduros têm uma vida útil muito curta. Fisiologicamente, a pitanga é uma fruta não climatérica, o que se caracteriza por apresentar alta taxa de respiração nos estágios iniciais de desenvolvimento, declinando ao longo da maturação. ${ }^{7,8} \mathrm{Com}$ isso, as estratégias de aproveitamento devem considerar esse aspecto do fruto com adoção de tecnologias que garantam perdas mínimas dos seus constituintes.

Ainda assim, o uso é muito limitado, deixando a exploração comercial de frutos restrita ao Nordeste do Brasil, onde são utilizados para produção de polpa e suco $^{10} \mathrm{~A}$ composição química dos frutos é altamente variável e depende de fatores como clima e condições do solo, manejo, nutrição das plantas, estádio de maturação dos frutos, variedade, entre outros. A composição (média de $77 \%$ de polpa e $23 \%$ de semente) é rica em cálcio, fósforo, 
antocianina e flavonoides, carotenoides e vitamina $C$, indicando sua alta propriedade antioxidante conforme a Tabela Brasileira de Composição dos Alimentos. ${ }^{11}$

Os atributos de qualidade variam conforme a maturação dos frutos, ${ }^{12,13}$ o que é determinante para o consumo dos mesmos. Assim, considerando que a maior parte das frutas nativas apresenta elevada variabilidade

\section{MATERIAL E MÉTODOS}

Frutos de pitanga foram colhidos apresentando coloração da casca variando de verde amarelada até completamente vermelha. A colheita foi realizada na zona rural, Sítio Paripe, localizada no município do Conde-PB, nas primeiras horas da manhã. Os frutos foram encaminhados para o Laboratório Multidisciplinar XI das Instituições Nova Esperança, João PessoaPB. Foi realizada uma seleção dos frutos e, com base na coloração da casca, separaramse três estádios de maturação (Figura 1). Para as avaliações, foram processados aproximadamente $250 \mathrm{~g}$ de frutos de cada estádio previamente definido. As sementes foram removidas manualmente e realizado o processamento dos frutos com casca e polpa.

A avaliação da coloração do epicarpo do fruto foi realizada objetivamente utilizando tanto nos atributos biométricos quanto na sua composição química, a caracterização da qualidade se torna a base das estratégias de agregação de valor a esses frutos que são ainda subutilizados. Desse modo, o objetivo deste trabalho é avaliar as mudanças na qualidade de frutos de pitangueira (Eugenia uniflora L.) durante a maturação.

o aplicativo Colorimeter (versão 1.6.6.2, Research Lab Tools, São Paulo, Brasil) instalado em um smartphone Android. O aplicativo permite a análise online e offline de amostras. A interface é fácil de usar e as mudanças de cor podem ser gravadas usando o recurso de câmera no smartphone. ${ }^{14}$ Usando o sistema de cor CIELab, as imagens foram capturadas com uma câmera de Smartphone Samsung Galaxy A50 (25 Mpx.). Foram tomadas quatro réplicas de cada ponto de referência. Em cada estádio de maturação, 10 frutos foram avaliados.

As leituras aconteceram sob luz branca com a câmera distante cerca de $10 \mathrm{~cm}$ da superfície do fruto. Os valores $L^{*}$ indicam luminosidade (preto $=0$ e branco $=100$ ); os valores $a^{*}$ indicam a variação de vermelhoverde (vermelho $a^{*}$ : 100 e verde $a^{*}$ : -100); os

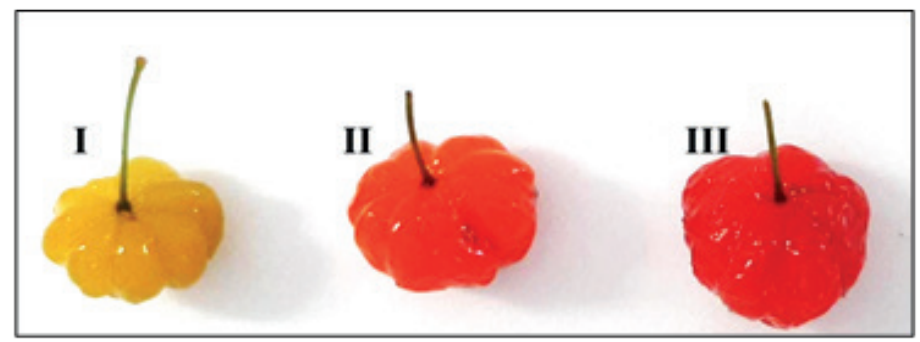

FIGURA 1: Frutos de pitangueira (Eugenia uniflora L.) colhidos nos estádios de maturação I: Amarelo DouradoAD, II: Laranja Avermelhado-LA e III: Completamente Vermelho-CV. João Pessoa-PB. 
valores de $b^{*}$ indicam a variação de amareloazul (amarelo $b^{*}$ : 100 e azul $b^{*}$ : -100). Croma (C) $\left(C=\left[\left(a^{*}\right) 2+\left(b^{*}\right) 2\right] 0,5\right)$ mede a saturação ou intensidade da cor e o ângulo Hue ( $\mathrm{h}=\operatorname{arc}$ $\left.\tan b^{*} / a^{*}\right)$ determina o vermelho, amarelo, verde, azul, roxo ou cores intermediárias entre pares adjacentes dessas cores básicas.

Determinações físico-químicas foram conduzidas: Sólidos solúveis (SS) - Determinado com refratômetro óptico Lorben, expressando-se o resultado em \%; Acidez Titulável (AT-g ácido cítrico $100 \mathrm{~g}^{-1}$ ) Determinada por titulometria com hidróxido de sódio 0,1 N, utilizando-se fenolftaleína a $1 \%$ como indicador; Relação SS/AT - Obtida pela divisão dos valores referentes aos sólidos solúveis e acidez titulável; pH: Determinado utilizando potenciômetro digital. Essas determinações seguiram as recomendações da Association of Official Analytical Chemistry - AOAC. ${ }^{15}$

\section{RESULTADOS E DISCUSSÃO}

Frutos de pitangueira(Eugenia uniflora L.) apresentaram diferenças que foram significativas $(p<0,01)$ entre os estádios de maturação avaliados tanto para os atributos de qualidade externos quanto aos internos. Os parâmetros da coloração da casca pelo sistema CIELab avaliados pelo aplicativo mostram que os frutos têm mudanças acentuadas ao longo de sua maturação (Tabela 1). O parâmetro L* (luminosidade) representa o brilho da casca e mostra que frutos de cor amarelada têm maiores valores $(A D \cong 76)$, reduzindo à medida que $a$ coloração vermelha predomina. Por sua vez, o parâmetro $a^{*}$ positivo aumentando com a maturação é uma resposta da crescente predominância do vermelho na casca. Frutos no estádio $\mathrm{CV}$ apresentaram valores $\cong 71$.
Foram determinados os conteúdos de flavonoides amarelos e antocianinas ( $\mathrm{mg}$ $100 \mathrm{~g}^{-1}$ ) por espectrofotometria de acordo com Francis ${ }^{16}$ com modificações. Os extratos foram preparados utilizando solução de álcool etílico (95\%). Para a determinação de flavonoides amarelos foram realizadas leituras a $374 \mathrm{~nm}$, para a de antocianinas, as amostras foram lidas a $535 \mathrm{~nm}$.

Foi admitido um delineamento experimental inteiramente casualizado com três repetições para as variáveis em que se avaliou o efeito da maturação nas características dos frutos. Para avaliação de coloração, cada fruto foi considerado como repetição. Os dados foram submetidos à análise de variância pelo teste $\mathrm{F}$ até um nível de significância de 5\% de probabilidade de erro e as médias foram comparadas pelo teste de Tukey. Foi utilizado o programa estatístico AgroEstat. ${ }^{17}$
Já o parâmetro $b^{*}$ apresentou valores decrescentes com a maturação em cerca de $30 \%$ do estádio $A D$ ao $C V$, indicado o desaparecimento da cor mais amarelada da casca. A intensidade da cor dos frutos nesses três estádios avaliados é intensa, aumentando em 14\% durante a maturação e o parâmetro $C$ (cromaticidade) indica que os frutos completamente vermelhos apresentaram altos valores. $O$ ângulo hue ${ }^{\circ} \mathrm{H}$ representa a variação da coloração que os frutos apresentaram. Com uma diferença de mais de $97 \%$ entre os estádios $A D$ e CV, confirma-se através dessa avaliação objetiva o que está posto na Figura 1. Os estádios LA e CV têm parâmetros de cor muito próximos, mantendo-se diferente estatisticamente nos parâmetros $a^{*} \mathrm{e}^{\circ} \mathrm{H}$. 
Durante o desenvolvimento dos frutos, a maturação é caracterizada por transformações físicas, químicas e bioquímicas que culminam no desenvolvimento do estádio mais palatável, ideal para o consumo. ${ }^{18} \mathrm{~A}$ coloração é o principal atributo da aparência externa do fruto da pitangueira, dado que cada estádio tem uma coloração característica. Consiste num importante índice de qualidade que se correlaciona com as mudanças da qualidade interna da polpa. A presença simultânea de carotenoides e compostos fenólicos (flavonoides amarelos e antocianinas) sintetizados com a maturação confere aos estádios suas características de coloração ${ }^{19,4}$ Nota-se que a utilização do aplicativo para smartphone Android Colorimeter (Research Lab Tools) é uma alternativa acessível para avaliação da qualidade desses frutos.

TABELA 1: Parâmetros objetivos de coloração (CIELab) da casca de frutos de pitangueira (Eugenia uniflora L.) em diferentes estádios de maturação (Amarelo Dourado-AD, Laranja Avermelhado-LA e Completamente VermelhoCV). João Pessoa-PB.

\begin{tabular}{cccccc}
\hline Estádios de Maturação & $\mathbf{L}^{*}$ & $\boldsymbol{a}^{*}$ & $\mathbf{b}^{*}$ & $\mathrm{C}$ & ${ }^{\circ} \mathrm{H}$ \\
\hline AD & $75,9 \pm 6,2 \mathrm{a}$ & $9,7 \pm 3,8 \mathrm{c}$ & $77,1 \pm 4,5 \mathrm{a}$ & $77,7 \pm 4,2 \mathrm{~b}$ & $44,0 \pm 1,8 \mathrm{a}$ \\
LA & $54,6 \pm 3,6 \mathrm{~b}$ & $68,1 \pm 0,7 \mathrm{~b}$ & $61,9 \pm 2,4 \mathrm{~b}$ & $92,1 \pm 1,1 \mathrm{a}$ & $10,3 \pm 2,7 \mathrm{~b}$ \\
CV & $47,3 \pm 1,3 \mathrm{~b}$ & $70,7 \pm 0,7 \mathrm{a}$ & $56,3 \pm 0,3 \mathrm{~b}$ & $90,4 \pm 0,5 \mathrm{a}$ & $0,9 \pm 1,0 \mathrm{c}$ \\
\hline \hline C.V. (\%) & 1,5 & 2,5 & 0,9 & 6,9 & 2,0 \\
\hline \hline
\end{tabular}

Médias com desvios padrões seguidas de mesma letra não diferem entre si pelo teste de Tukey a $5 \%$ de probabilidade de erro.

A acidez dos frutos da pitangueira apresentou uma redução nos valores médios do estádio $A D$ para $0 \mathrm{CV}$, de 3,1 para 2,3 $\mathrm{g}$ $100 \mathrm{~g}^{-1}$ (Figura 2A). Resposta em função da maturação também pode ser observada para $\mathrm{o} \mathrm{pH}$, onde frutos mais maduros têm um $\mathrm{pH}$ mais elevado (Figura 2D). Por sua vez, o conteúdo de sólidos solúveis (SS) na pitanga apresentou uma diferença significativa entre os estádios de maturação, onde se observou um aumento de $\cong 30 \%$ (Figura $2 \mathrm{~B}$ ). A relação SS/AT é um indicativo da palatabilidade dos frutos que, na pitanga, aumenta com a variação da coloração da casca. $O$ decréscimo da acidez titulável paralelo ao aumento do conteúdo de sólidos solúveis leva os frutos do estádio $\mathrm{CV}$ a terem os maiores valores observados (Figura 2C). A pitanga é uma fruta de sabor característico marcado por uma acidez elevada, mesmo em estádios de maturação completamente maduros. ${ }^{20}$ Considerando que é raramente encontrada no mercado como fruta fresca, a pitanga é mais comumente encontrada na forma processada, como sucos, polpa congelada e geleias. $^{10}$ Com isso, há alterações na composição da polpa antes e depois do processamento.

Bagetti et al. $^{19}$ avaliaram características físico-químicas de pitangas de polpa roxa, vermelha e alaranjada oriundas de lotes de plantas distintas. Observaram que os atributos físico-químicos se diferenciam dependendo da cor. Pitangas vermelhas apresentaram valores de $\mathrm{pH}$, SS e AT inferiores aos reportados nesta pesquisa. Já Santos et al. $^{20}$ reportaram valores desses atributos de qualidade para pitangas vermelhas armazenadas sob atmosfera modificada e diferentes temperaturas. Foi verificado que a refrigeração é um fatorimportante na conservação da qualidade e que estádios intermediários (vermelhoalaranjado) são mais indicados. 


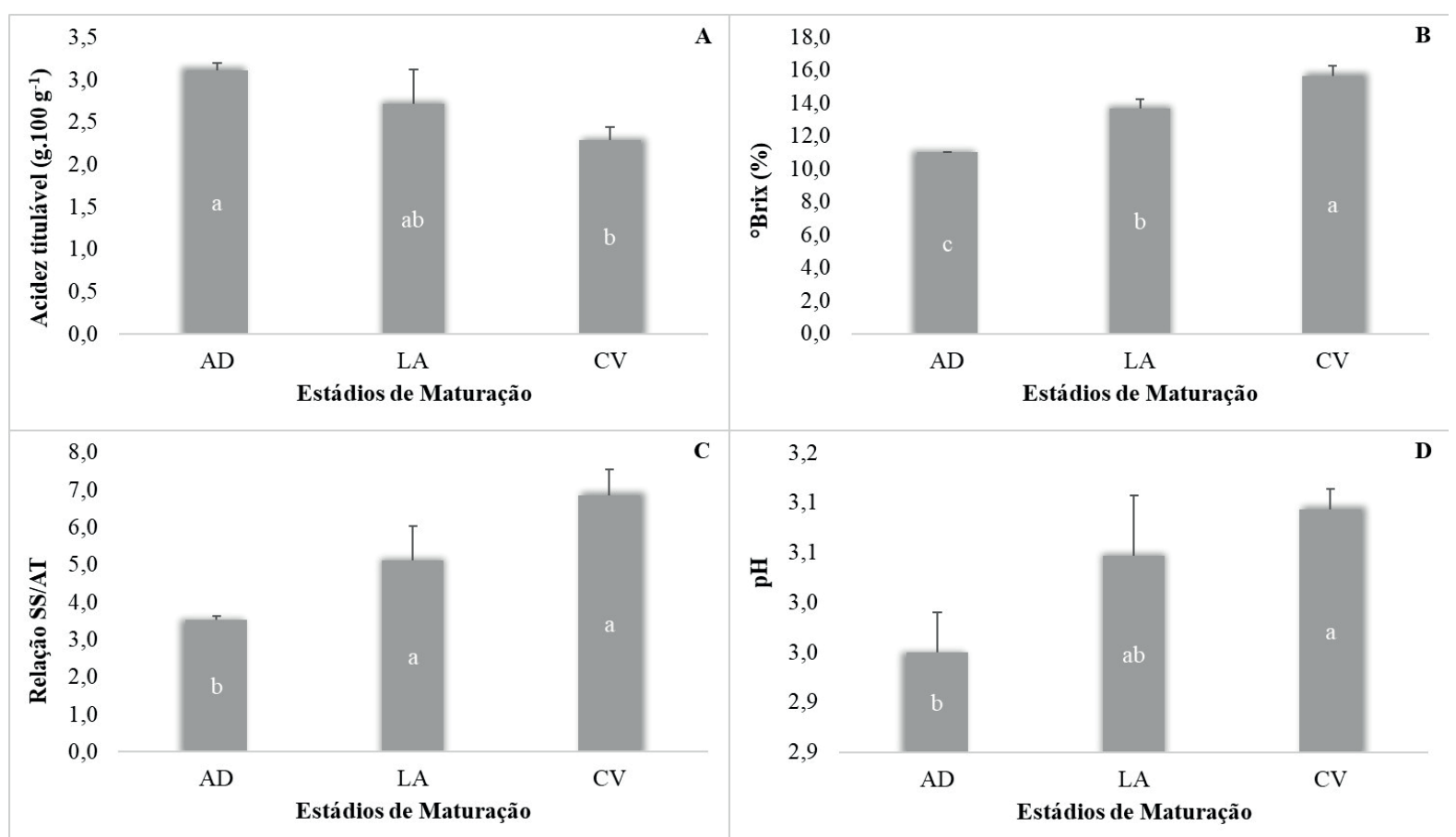

FIGURA 2: Atributos físico-químicos de frutos de pitangueira (Eugenia uniflora L.) em diferentes estádios de maturação (Amarelo Dourado-AD, Laranja Avermelhado-LA e Completamente Vermelho-CV). João Pessoa-PB. (A: Acidez titulável; B: Sólidos Solúveis; C: Relação SS/AT; D: pH). Barras apresentando letras iguais não diferem entre si pelo teste de Tukey a $5 \%$ de probabilidade. Barras verticais indicam o desvio padrão da média.

Os valores de $\mathrm{pH}$, SS e AT reportados nessas condições estão coerentes aos observados aqui. Esses atributos de qualidade são determinantes para o sabor e as mudanças observadas são decorrentes de transformações caracte-rísticas da maturação. $7,8,21^{3}$

Diversas transformações durante a maturação afetam não apenas as características físicas, mas também as físicoquímicas. Nesta fase, as modificações são irreversíveis e decorrentes de processos bioquímicos e fisiológicos, levando, sobretudo a modificações na taxa respiratória, degradação da clorofila em paralelo com a biossíntese de carotenoides, antocianinas e os outros componentes responsáveis pela cor, sabor e aroma. ${ }^{18,21}$ Acompanhar essas transformações em frutas nativas de alto potencial como a pitanga é essencial para o conhecimento da biologia da espécie e contribuir para a valorização de espécies da biodiversidade brasileira.

$\mathrm{Na}$ pitanga, a coloração da casca e da polpa consiste numa das mais acentuadasmudanças que ocorrem na maturação. O conteúdo de flavonoides amarelos e de antocianinas nos frutos aumentaram do estádio $A D$ para $O C V$, sobretudo para as antocianinas cujo conteúdo aumentou em mais de $88 \%$ (Figura 3). Essas duas classes fazem parte do perfil de compostos bioativos presentes não somente na casca, mas também na polpa da pitanga em quantidades elevadas, estando bem acima do que é observado para outras frutas tropicais como cajá (Spondias mombin L.) maracujá (Passiflora edulis Sims) tamarindo (Tamarindo indica L.) e acerola (Malpighia emarginata D.C.). ${ }^{2}$ Além disso, devido à variabilidade genética, pitangas completamente maduras apresentam padrões distintos de pigmentos 
na casca. Denardin et al. ${ }^{22}$ observaram que linhagens de pitanga de polpa laranja, vermelha e roxa apresentam quantidades de compostos fenólicos bem distintas, em que a linhagem roxa apresentou um conteúdo superior, $43 \%$ em relação à laranja e $46 \%$ em relação à vermelha, se configurando uma fruta rica em compostos bioativos de alta atividade antioxidante.

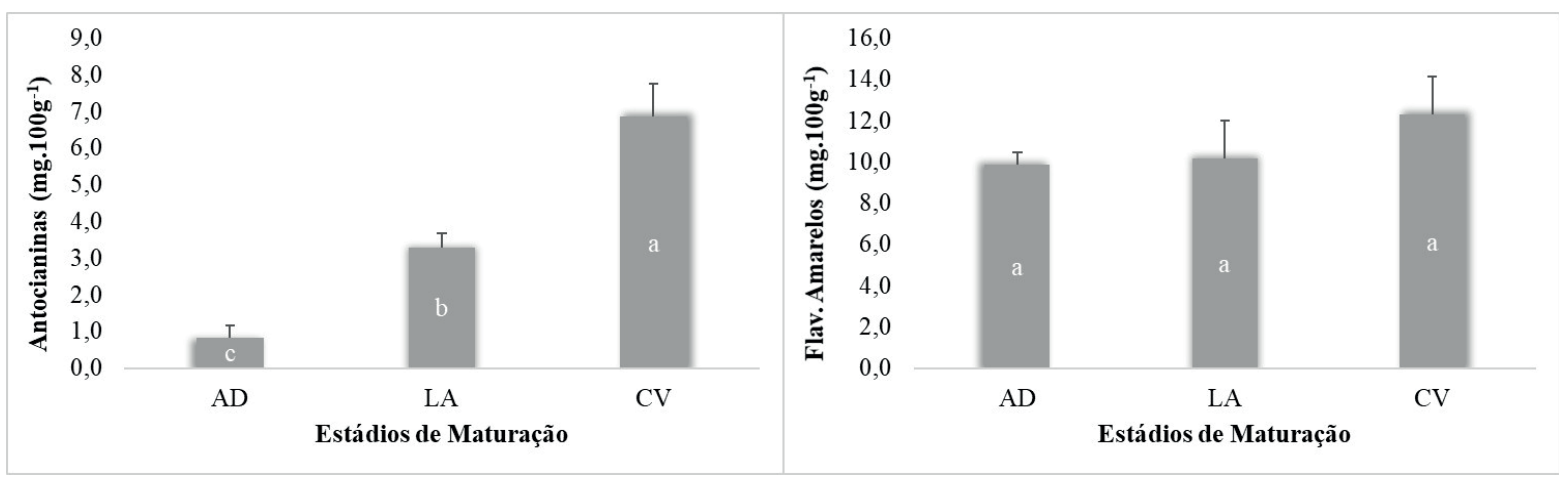

FIGURA 2: Conteúdos de Flavonoides amarelos (A) e Antocianinas (B) de frutos de pitangueira (Eugenia uniflora L.) em diferentes estádios de maturação (Amarelo Dourado-AD, Laranja Avermelhado-LA e Completamente Vermelho-CV). João Pessoa-PB. Barras apresentando letras iguais não diferem entre si pelo teste de Tukey a $5 \%$ de probabilidade. Barras verticais indicam o desvio padrão da média.

Várias espécies do gênero Eugenia são apontadas como fontes de compostos benéficos para a saúde, apresentando potencial terapêutico para serem utilizadas no tratamento do diabetes e suas comorbidades. ${ }^{23} \mathrm{O}$ aumento da concentração desses metabólitos ao longo da maturação é bem reportado na literatura. São sintetizados através da via dos fenilpropanoides e, por serem metabólitos secundários com diversas funções protetoras, sua síntese é regulada por vários sinais de estresse e fatores ambientais. Desse modo, práticas pré e pós-colheita podem afetar o acúmulo ou a degradação desses compostos em frutas. Temperatura, propriedades do solo, irradiação luminosa, irrigação, fertilizantes, estádio de maturação são condições pré-colheita que levam a modificações no perfil qualiquantitativo desses compostos. ${ }^{24}$

\section{CONCLUSÃO}

Frutos de pitangueira (Eugenia uniflora L.) vermelha apresentam valores elevados para a acidez titulável e sólidos solúveis no estádio completamente vermelho (CV). O mesmo ocorreu para o conteúdo de antocianinas e flavonoides amarelos.
Dentre os atributos físicos, o efeito mais acentuado da maturação foi observado para os parâmetros de cor $a^{*} \mathrm{e}^{\circ} \mathrm{H}$, avaliados pelo aplicativo para smartphone; já SS, relação SS/AT e antocianinas foram os atributos físico-químicos com diferenças mais expressivas. 


\section{REFERÊNCIAS BIBLIOGRÁFICAS}

1. Chang SK, Alasalvar C, Shahidi F. Superfruits: Phytochemicals, antioxidant efficacies, and health effects-A comprehensive review. Crit Rev Food Sci Nutr. 2018; 59(10): 1580-1604.

2. Silva LMR, Figueiredo EAT, Ricardo, NMPS, Vieira, IGP, Figueiredo, RW, Brasil, I M, Gomes, CL. Quantification of bioactive compounds in pulps and by-products of tropical fruits from Brazil. Food Chem. 2014; 143: 398-404. doi:10.1016/j.foodchem.2013.08.

3. Yahia EM, Maldonado CME, Svendsen, M. The contribution of fruit and vegetable consumption to human health. Fruit and vegetable Phytochemicals. Yahia, EM, ed. Hoboken: John Wiley \& Sons, 2017; 3-52.

4. Pereira E, Raphaelli CO, Radünz M, Camargo TM, Vizzotto M. Biological activity and chemical composition of native fruits. Agrocienc Urug, 2021; 25(E2): e815-e815.

5. Blancke, R. Tropical fruits and other edible plants of the world: An illustrated guide. Cornell University Press. 2016.

6. Bezerra J, Lira Junior JS, Silva Junior, JF. Eugenia uniflora: pitanga. Embrapa Tabuleiros CosteirosCapítulo em livro científico (ALICE), 2018.

7. Lorenzi H, Bacher L, Lacerda M, Sartori S. Frutas Brasileiras e Exóticas Cultivadas, São Paulo, Editora Plantarum Ltda, 2006.

8. Santos AF, Silva SM, Mendonca RMN, Alves RE, Filgueiras HAC. Maturation and Ripening Changes in Surinam Cherry Eugenia uniflora L.). Proceedings of the Tropical Region - American Society for Horticultural Science, Morelos, México. 2002; 45: 4547.

9. Vizzotto M, Cabral L, Santos A. Pitanga (Eugenia uniflora L.). In: Postharvest biology and technology of tropical and subtropical fruits. Woodhead Publishing, 2011. p. 272-288e.
10. Franzon, R. C., Carpenedo, S., Viñoly, M. D., \& do CB Raseira, M. (2018). Pitanga-Eugenia uniflora L. In Exotic Fruits (pp. 333-338). Academic Press.

11. UNICAMP-Universidade de Campinas, 2011. Tabela Brasileira de composição dos alimentos. 4 ed. Campinas. Disponível em: http://www.unicamp. br/ nepa/taco/contar/taco_4_edicao_ampliada_e_revisada. Acesso em outubro de 2015.

12. Chaves Neto JRC, Santos LF, Dantas AL, Dantas RL, Schunemann APP, Silva SM. Qualidade de Frutos de Acessos de Cajá-Mangueira Durante a Maturação. B.CEPPA. 2019; 36(1): 39-54.

13. Chaves Neto JRC, Silva SM, Dantas, RL. Atributos de qualidade, compostos bioativos e atividade antioxidante de frutos de uvaieira durante a maturação. Agrarian. 2020; 13(49), 269-308.

14. Ravindranath R, Periasamy AP, Roy P, Chen YW, Chang HT. Smart app-based on-field colorimetric quantification of mercury via analyte-induced enhancement of the photocatalytic activity of TiO2Au nanospheres. Anal. Bioanal. Chem., 2018; 410: 4555-64.

15. Horwitz W, Latimer GW. Association of Official Analytical Chemistry-AOAC. Official Methods of Analysis (18th ed.). Gaithersburg: AOAC International, 2007.

16. Francis FJ. Analysis of anthocyanins. In P. Markakis (Ed.), Anthocyanins as food colors. New York: Academic Press. 1982; 181-207.

17. Barbosa JC, Maldonado Júnior, W. Experimentação Agronômica e AGROESTAT: Sistema Para Análises Estatísticas De Ensaios Agronômicos. Jaboticabal: Multipress, 2015.

18. Paliyath G, Murr DP. Biochemistry of Fruits. In: Paliyath G, Murr DP, Handa AK, Lurie S. (eds) Postharvest Biology and Technology of Fruits, 
Vegetables and Flower. Wiley-Blackwell Publishing, cap.3, 2008; 19-50.

19. Bagetti M. Physicochemical Characterization and Antioxidant Capacity of Pitanga Fruits (Eugenia uniflora L.). Food Sci Technol Int. 2011; 31: 147-54. 20. Santos AF, Silva SM, Alves RE. Armazenamento de pitanga sob atmosfera modificada e refrigeração: I-transformações químicas em pós-colheita, Rev Bras Frutic, 2006; 28(1): 36-4.

21. Dantas AL, Silva SM, Dantas RL, Sousa ASB; Schunemann APP. Desenvolvimento, fisiologia da maturação e indicadores do ponto de colheita de frutos da umbugueleira (Spondias sp.). Rev Bras Frutic. 2016; 38(1): 33-42.
22. Denardin CC, Hirsch GE, Rocha RF, Vizzotto $M$, Henriques AT, Moreira, JCF et al. Antioxidant capacity and bioactive compounds of four Brazilian native fruits. J Food Drug Anal. 2015; 23(3): 387-98.

23. Araujo NMP, Arruda HS, Farias DP, Molina G, Pereira GA, Pastore GM. Plants from the genus Eugenia as promising therapeutic agents for the management of diabetes mellitus: A review. Food Res. Int. 2021; 142, 110182.

24. Rosa LA, Moreno-Escamilla JO, RodrigoGarcía J, Alvarez-Parrilla E. Phenolic Compounds. Postharvest Physiology and Biochemistry of Fruits and Vegetables. 2019; 253-271. doi:10.1016/b978-0-12813278-4. 